\title{
RATE-DISTORTION PROBLEM FOR PHYSICS BASED DISTRIBUTED SENSING
}

\author{
Baltasar Beferull-Lozano, Robert L. Konsbruck, Martin Vetterli \\ School of Computer and Communication Sciences, \\ Swiss Federal Institute of Technology-EPFL, CH-1015 Lausanne, Switzerland, \\ E-mail: \{Baltasar.Beferull, Robert.Konsbruck, Martin.Vetterli\}@epfl.ch
}

\begin{abstract}
We consider the rate-distortion problem for sensing the continuous space-time physical temperature in a circular ring on which a heat source is applied over space and time, and which is allowed to cool by radiation or convection. The heat source is modelled as a continuous space-time stochastic process which is bandlimited over space and time. The temperature field is the result of a certain continuous space-time convolution of the heat source with the Green's function corresponding to the heat equation, which is space and time invariant. The temperature field is sampled at uniform spatial locations by a set of sensors and it has to be reconstructed at a base station. The goal is to minimize the meansquare-error per second, for a given number of nats per second, assuming ideal communication channels between sensors and base station. We find a) the centralized $R^{\mathrm{c}}(D)$ function of the temperature field, where the base station can optimally encode all the space-time samples jointly. Then, we obtain b) the $R^{s-i}(D)$ function, where each sensor, independently, encodes its samples optimally over time and c) the $R^{\mathrm{st}-\mathrm{i}}(D)$ function, where each sensor is constrained to encode also independently over time. We also study two distributed prediction-based approaches: a) with perfect feedback from the base station, where temporal prediction is performed at the base station and each sensor performs differential encoding, and b) without feedback, where each sensor locally performs temporal prediction.
\end{abstract}

\section{INTRODUCTION}

In sensor networks, some continuous space-time physical phenomenon is sampled by a set of remote sensors, and an estimate of this physical phenomenon has to be obtained at a base station. Due to the restricted processing power in the sensors, it is usually not allowed to have communication between sensors, and it is very important to minimize the rate at which the information is encoded. There has been important research work going in this direction [2]. However, in all this previous work, the physics that describes the phenomenon has not been taken into account.

In this paper, we incorporate the physics into a sensor network rate-distortion problem where the network is sensing the continuous space-time physical temperature on a closed circular ring on which a heat source is applied over space and time, and which is allowed to cool by electromagnetic radiation or convection. The heat source is modelled as a continuous Gaussian space-time (widesense stationary) stochastic process which is bandlimited over space

The work presented in this paper was supported (in part) by the National Competence Center in Research on Mobile Information and Communications Systems (NCCR-MICS), a center supported by the Swiss National Science Foundation under grant number 5005-67322.

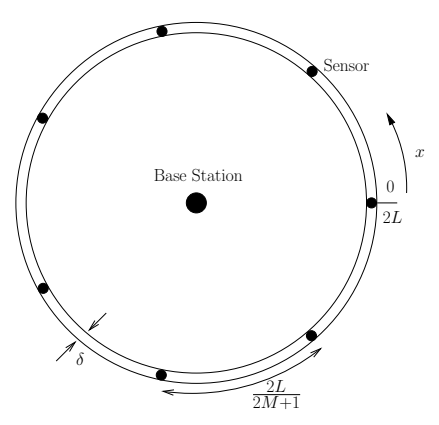

Fig. 1. Ring structure.

and time. The temperature field over the ring is the result of a certain continuous space-time convolution of the heat source with the Green's function of our system, which turns out to be space and time invariant. The temperature field is sampled at uniform spatial locations by a set of sensors and the goal is to minimize the distortion measured in mean-square-error per second at a base station, for a given number of nats per second, assuming ideal communication channels between the sensors and the base station.

This paper is structured as follows. In Section 2, we explain the physics governing the temperature field on a ring, and the concept of Green's function of the system. In Section 3, we explain the rate-distortion problem. In Section 4, we find analytically the centralized $R^{\mathrm{c}}(D)$ function, where the base station can encode all the spatio-temporal samples together, and in Section 5, we obtain a) the $R^{\text {s-i }}(D)$ function, where each sensor, independently, encodes its samples optimally over time, and b) the $R^{\mathrm{st}-\mathrm{i}}(D)$ function, where each sensor encodes also independently over time. Then, in Section 6, we study two distributed prediction-based approaches: a) with perfect feedback from the base station, where temporal prediction is performed at the base station and each sensor performs differential encoding, and b) without feedback, where each sensor locally performs temporal prediction. We also explain briefly nested code based constructions [4] for our problem.

\section{TEMPERATURE PROBLEM IN THE RING}

The sensed physical field that we consider in this work is the continuous space-time temperature field $T(x, t)$ in a closed circular (heat conductor) ring of length $2 L$, where $x \in[0,2 L[$ indicates the spatial position in the ring (see Fig. 1), and $t \in \mathbb{R}$. It is assumed that the ring has a very small cross-section, so that the temperature at all points of the cross-section may be taken to be the same. A space-time varying heat source (e.g. electric heating), measured in (Watts/meter ${ }^{3}$ ), is applied on the ring, which is also 
allowed to cool by electromagnetic radiation or forced convection to its surrounding medium.

\subsection{Heat Source and Thermal Properties}

The heat source $g(x, t)$ is allowed to both produce (additive source) and withdraw (sink) heat, and we model it by a continuous spacetime Gaussian stochastic process, which is periodic and bandlimited over space, and which is bandlimited over time, that is:

$g(x, t)=\frac{g_{0}(t)}{\sqrt{2}}+\sum_{m=1}^{M}\left(g_{m}(t) \cos \left(\lambda_{m} x\right)+g_{m+M}(t) \sin \left(\lambda_{m} x\right)\right)$

where $M+1$ is the number of harmonics (spatial bandwidth of $2 M+1$ ), $\lambda_{m}=\frac{m \pi}{L}, m \geq 0$ (thus the fundamental spatial period is $2 L$, which is equal to the length of the ring), and $\left\{g_{m}(t)\right\}_{m=0}^{2 M}$ is a set of $2 M+1$ continuous-time real wide-sense stationary (WSS) Gaussian stochastic processes with zero mean and which are assumed to be independent, that is, the cross-correlation $R_{g_{m_{1}} g_{m_{2}}}(\gamma)=$ $E\left[g_{m_{1}}(t+\gamma) g_{m_{2}}(t)\right]=0$ for $m_{1} \neq m_{2}, \forall \gamma \in \mathbb{R}$. Moreover, each continuous-time process $g_{m}(t), m=0, \ldots, 2 M$, is assumed to be bandlimited to $[-\pi, \pi]$ and to have a constant gain $G_{m}>0$, that is, each process has a power spectral density (PSD) given by $S_{g_{m}}(\Omega)=G_{m} 1_{[-\pi, \pi]}(\Omega)$, where $\Omega$ denotes the continuoustime angular frequency. We also assume ${ }^{1}$ that $G_{m}=G_{m+M}$, for $m=1, \cdots, M$.

The thermal parameters [1] of the ring, which play an important role in the rate-distortion properties, are: a) its thermal conductivity $\kappa$ in (Watts/(meter Kelvin)), b) its thermal diffusivity $\alpha=\kappa /\left(\rho C_{p}\right)$ in (meters ${ }^{2} /$ second), where $\rho$ is the density in (kg/meters ${ }^{3}$ ) and $C_{p}$ is the specific heat in (Joules/(kg Kelvin)), and c) its dissipation parameter $\mu=\frac{2 h}{\kappa \delta}$ in $\left(\right.$ meters $^{-2}$ ), where $h$ is the heat transfer coefficient and $\delta$ is the thickness of the ring.

\subsection{Heat Differential Equation for the Ring}

Given a source $g(x, t)$ and the different thermal parameters of the ring, the differential equation describing the resulting temperature $T(x, t)$ in the ring, is given by (see Fig. 1 ):

$$
\left.\begin{array}{c}
\frac{\partial^{2} T(x, t)}{\partial x^{2}}+\frac{g(x, t)}{\kappa}-\mu T(x, t)=\frac{1}{\alpha} \frac{\partial T(x, t)}{\partial t} \\
T(0, t)=T(2 L, t) \\
\left.\frac{\partial T(x, t)}{\partial x}\right|_{x=0}=\left.\frac{\partial T(x, t)}{\partial x}\right|_{x=2 L}
\end{array}\right\} \text { (periodic boundary conditions) }
$$

Using Green's Theorem [1], the solution of (2) is given by:

$$
T(x, t)=\frac{\alpha}{\kappa} \int_{\tau=-\infty}^{\tau=t} \int_{\xi=0}^{\xi=2 L} g(\xi, \tau) h(x-\xi, t-\tau) d \xi d \tau
$$

where $h(x, t ; \xi, \tau)=h(x-\xi, t-\tau)$ is the Green's function of the system described by (2), which turns out to be time and space invariant. This means that the operator corresponding to the differential equations in (2) is equivalent to a linear time-invariant space-invariant system described by an impulse response $h(x, t)$. This impulse response can be shown to be [1]:

$$
h(x, t)=u(t)\left(\frac{1}{2 L} e^{-\mu \alpha t}+\frac{1}{L} \sum_{m=1}^{\infty} e^{-\left(\lambda_{m}^{2}+\mu\right) \alpha t} \cos \left(\lambda_{m} x\right)\right)
$$

${ }^{1}$ This condition is necessary to make $T(x, t)$ stationary over space. where $u(t)$ is the step function, that is, $u(t)=1$ for $t \geq 0$ and $u(t)=0$ for $t<0$. Thus, the impulse response is causal over time and periodic over space with period $2 L$, which implies that there is a continuous-space circular convolution and the final temperature process $T(x, t)$, which is periodic over space, is given by:

$$
\begin{aligned}
& T(x, t)=\frac{\beta_{0}(t)}{\sqrt{2}}+\sum_{m=1}^{M}\left(\beta_{m}(t) \cos \left(\lambda_{m} x\right)+\beta_{m+M}(t) \sin \left(\lambda_{m} x\right)\right) \\
& \beta_{m}(t)=g_{m}(t) * h_{m}(t), \quad h_{m}(t)=u(t)\left(\frac{\alpha}{\kappa} e^{-\left(\lambda_{m}^{2}+\mu\right) \alpha t}\right)
\end{aligned}
$$

where $\lambda_{m}=\lambda_{m+M}$ for $m=1, \cdots, M$, and the $2 M+1$ independent Gaussian processes $\left\{\beta_{m}(t)\right\}_{m=0}^{2 M}$ are still bandlimited to $[-\pi, \pi]$, but their spectral densities are given by:

$$
\begin{aligned}
& S_{\beta_{m}}(\Omega)=\mathcal{F}\left(h_{m}(t)\right) G_{m} 1_{[-\pi, \pi]}(\Omega)=\frac{A_{m} c_{m}^{2}}{\Omega^{2}+c_{m}^{2}}, \quad|\Omega| \leq \pi, \\
& A_{m}=\frac{G_{m}}{\kappa^{2}\left(\lambda_{m}^{2}+\mu\right)^{2}}, \quad c_{m}=\left(\lambda_{m}^{2}+\mu\right) \alpha
\end{aligned}
$$

\section{RATE-DISTORTION PROBLEM}

The temperature process is sampled uniformly in space and time at the corresponding spatial and temporal Nyquist sampling frequencies, respectively. This means that the sampling period in space is $T_{s}=\frac{2 L}{2 M+1}$ meters and the sampling period in time is $T_{t}=1$ second. The sensing task is performed by $2 M+1$ sensors which are located at $x_{n}=\frac{2 L}{2 M+1} n, n=0, \ldots, 2 M$. Over time, the $n$-th sensor takes the samples $\left\{T\left(x_{n},-\infty\right), \ldots, T\left(x_{n}, t_{j}\right), T\left(x_{n}, t_{j+1}\right)\right.$, $\left.\ldots, T\left(x_{n}, \infty\right)\right\}$, where $t_{j+1}-t_{j}=1$.

The continuous space-time temperature process $T(x, t)$ has to be reconstructed at a base station (BS) located at the center of the ring, as illustrated in Fig. 1. Given a reconstructed temperature process $\widehat{T}(x, t)$, the distortion, which we denote by $D$, is measured in mean-square-error (MSE) per second, and is defined by:

$$
D=\frac{1}{2 L} \int_{0}^{2 L} \lim _{\mathbb{T} \rightarrow \infty} \frac{1}{2 \mathbb{T}} \int_{-\mathbb{T}}^{\mathbb{T}} E\left[(T(x, t)-\widehat{T}(x, t))^{2}\right] \quad d t d x
$$

The goal is to minimize the distortion at the BS for a given total number of nats per second, denoted by $R$. Regarding the communication model, it is assumed throughout this work that a) from the sensors to the BS, there are ideal channels, that is, if the $n$-th sensor has to deliver $R_{n}$ nats per second to the $\mathrm{BS}$, the sensor will spend the necessary power to transmit them without error ${ }^{2}$, and $b$ ) from the BS to the sensors, there is a perfect channel where the BS can transmit at an infinite rate to the sensors (perfect feedback). Our objective is to study the rate-distortion problem under these communication channel idealizations.

\section{4. $R^{\mathrm{C}}(D)$ FOR CENTRALIZED CODING}

In this section, we obtain the rate-distortion function for the most idealized case where the BS receives all the (unquantized) spacetime samples $\left\{T\left(x_{n},-\infty\right), \ldots, T\left(x_{n}, \infty\right)\right\}_{n=0}^{2 M}$ and encodes all of them jointly. We denote this function by $R^{\mathrm{c}}(D)$. In order to find $R^{\mathrm{c}}(D)$, we first express the distortion $D$, as defined in (4), as a function of the distortions corresponding to the processes $\left\{\beta_{m}(t)\right\}_{m=0}^{2 M}$, which completely determine the temperature process $T(x, t)$. Let $\boldsymbol{F}$ be the $(2 M+1) \times(2 M+1)$ matrix with $m$ th row $(\boldsymbol{F})_{m}=\left[\frac{1}{\sqrt{2}}, \cos \left(\frac{2 \pi m}{2 M+1}\right), \cdots, \cos \left(\frac{2 \pi m M}{2 M+1}\right), \sin \left(\frac{2 \pi m}{2 M+1}\right)\right.$, $\left.\cdots, \sin \left(\frac{2 \pi m M}{2 M+1}\right)\right]$. Let $\boldsymbol{\beta}(t)=\left[\beta_{0}(t), \ldots, \beta_{2 M}(t)\right]^{T}$ and $\boldsymbol{T}(t)=$

\footnotetext{
${ }^{2}$ The consideration of a real channel is a subject of our current research.
} 
$\left[T(0, t), \ldots, T\left(2 M \frac{2 L}{2 M+1}, t\right)\right]^{T}$, which is the spatial vector of temperature samples. Then, the BS can find $\boldsymbol{\beta}(t)$ from $\boldsymbol{T}(t)$ by:

$$
\boldsymbol{T}(t)=\boldsymbol{F} \boldsymbol{\beta}(t) \Longleftrightarrow \boldsymbol{\beta}(t)=\frac{2}{2 M+1} \boldsymbol{F}^{T} \boldsymbol{T}(t)
$$

Thus, to provide a set of reconstructed processes $\left\{\widehat{\beta}_{m}(t)\right\}_{m=0}^{2 M}$ is equivalent to providing a reconstruction $\widehat{T}(x, t)$. Because of the orthogonality property of the Fourier series over space, and the fact that $\left\{\beta_{m}(t)\right\}_{m=0}^{2 M}$ are independent random processes, it can be easily shown that $D=\frac{1}{2} \sum_{m=0}^{2 M} D_{m}$ and $R=\sum_{m=0}^{2 M} R_{m}$, where $D_{m}$ is the MSE per second associated to the temporal process $\beta_{m}(t)$, which is given by $D_{m}=\lim _{\mathbb{T} \rightarrow \infty} \frac{1}{2 \mathbb{T}} \int_{-\mathbb{T}}^{\mathbb{T}} E\left[\left(\beta_{m}(t)-\right.\right.$ $\left.\left.\widehat{\beta}_{m}(t)\right)^{2}\right] d t$, and $R_{m}$ is the corresponding number of nats per second. These additivity properties allow us to use the equalslope technique [3] over the set of processes $\left\{\beta_{m}(t)\right\}_{m=0}^{2 M}$. In order to do this, we first need to calculate the rate-distortion functions $\left\{R_{m}\left(D_{m}\right)\right\}_{m=0}^{2 M}$. Notice that since each process $\beta_{m}(t)$ is bandlimited, each $R_{m}\left(D_{m}\right)$ can be calculated by considering the discrete-time sampled process $\beta_{m}\left(t_{j}\right)$ and performing waterfilling over time [3], that is, $R_{m}\left(D_{m}\right)$ is given by:

$$
\begin{aligned}
& D_{m}\left(\theta_{m}\right)=\frac{1}{2 \pi} \int_{-\pi}^{\pi} \min \left[\theta_{m}, S_{\beta_{m}}(\omega)\right] d \omega \\
& R_{m}\left(\theta_{m}\right)=\frac{1}{4 \pi} \int_{-\pi}^{\pi} \max \left[0, \log \left(\frac{S_{\beta_{m}}(\omega)}{\theta_{m}}\right)\right] d \omega
\end{aligned}
$$

where $\theta_{m} \in\left[0, S_{\beta_{m}}(0)\right]$ is the waterfilling parameter, and $\omega$ denotes the discrete-time angular frequency. Performing this calculation, we get the following parameterized expression for $R_{m}\left(D_{m}\right)$ :

$$
\begin{aligned}
& D_{m}\left(\phi_{m}\right)=\frac{A_{m} c_{m}}{\pi}\left(\arctan \left(\frac{\pi}{c_{m}}\right)+\frac{\phi_{m}}{1+\phi_{m}^{2}}-\arctan \left(\phi_{m}\right)\right) \\
& R_{m}\left(\phi_{m}\right)=\frac{c_{m}}{\pi}\left(\phi_{m}-\arctan \left(\phi_{m}\right)\right) \\
& \quad \text { for } 0 \leq \phi_{m} \leq \frac{\pi}{c_{m}} \\
& D_{m}\left(\phi_{m}\right)=\frac{A_{m}}{1+\phi_{m}^{2}} \\
& \quad R_{m}\left(\phi_{m}\right)=\frac{1}{2} \log \left(\frac{\left(1+\phi_{m}^{2}\right) c_{m}^{2}}{c_{m}^{2}+\pi^{2}}\right)+1-\frac{c_{m}}{\pi} \arctan \left(\frac{\pi}{c_{m}}\right) \\
& \quad \text { for } \phi_{m} \geq \frac{\pi}{c_{m}}
\end{aligned}
$$

where $\phi_{m}=\sqrt{\frac{A_{m}-\theta_{m}}{\theta_{m}}}$, and the parameters $A_{m}$ and $c_{m}$ are as defined in (3). Using this, we can show the following Theorem:

Theorem 1 The $R^{c}(D)$ function is given by:

$$
D^{c}(\eta)=\frac{1}{2} \sum_{m=0}^{2 M} D_{m}\left(f_{m}(\eta)\right), R^{c}(\eta)=\sum_{m=0}^{2 M} R_{m}\left(f_{m}(\eta)\right)
$$

where $f_{m}(\eta)=\sqrt{2 A_{m} \eta-1}$ and $\eta \geq \min _{\{0 \leq m \leq 2 M\}} \frac{1}{2 A_{m}}$. Proof: See [4].

\section{RATE DISTORTION FOR LOCAL CODING}

\section{1. $R^{\mathrm{s}-\mathrm{i}}(D)$ Function for Spatially Independent Coding}

First, we consider the case where each sensor (independently from the other sensors) at its position $x_{n}=2 L n /(2 M+1)$ observes the temporal process $T\left(x_{n}, t\right)$, encodes it by optimally exploiting the temporal correlation, and transmits the encoded process to the BS. It can be shown that the original temperature $T(x, t)$ is completely determined by the spatial samples according to:

$$
T(x, t)=\sum_{n=0}^{2 M} T\left(x_{n}, t\right) f_{n}(x)
$$

where $f_{n}(x)=\frac{2}{2 M+1}\left(\frac{1}{2}+\sum_{m=1}^{M}\left(\cos \left(m n \frac{2 \pi}{2 M+1}\right) \cos \left(\lambda_{m} x\right)+\right.\right.$ $\left.\left.\sin \left(m n \frac{2 \pi}{2 M+1}\right) \sin \left(\lambda_{m} x\right)\right)\right)$. Receiving the quantized spatial samples of the temperature process, the BS reconstructs an estimate $\widehat{T}(x, t)$ using (7). We call $R^{s-i}(D)$ the rate distortion function corresponding to this scenario. Since it can be shown that the family $\left\{f_{n}(x)\right\}_{n=0}^{2 M}$ satisfies orthogonality, and because of the constraint of the source coding to be performed locally, it follows that $D^{\mathrm{s}-\mathrm{i}}=\frac{1}{2 M+1} \sum_{n=0}^{2 M} D_{n}$ and $R^{\mathrm{s}-\mathrm{i}}=\sum_{n=0}^{2 M} R_{n}$, where $D_{n}$ is the MSE per second associated to the temporal process $T\left(x_{n}, t\right)$ and $R_{n}$ is the rate used by the encoder of the sensor located at position $x_{n}$. Notice that the power spectral density of each locally observed process $T\left(x_{n}, t\right)$ does not depend on the location $x_{n}$. Let $S(\omega)$ denote this power spectral density. The rate distortion function $R^{\mathrm{s}-\mathrm{i}}(D)$ is then given by the following Theorem:

Theorem 2 The $R^{s-i}(D)$ function is given by:

$$
\begin{aligned}
D^{s-i}(\theta) & =\frac{1}{2 \pi} \int_{-\pi}^{\pi} \min [\theta, S(\omega)] d \omega \\
R^{s-i}(\theta) & =\frac{2 M+1}{4 \pi} \int_{-\pi}^{\pi} \max \left[0, \log \left(\frac{S(\omega)}{\theta}\right)\right] d \omega
\end{aligned}
$$

where $S(\omega)=\frac{1}{2} \frac{A_{0} c_{0}^{2}}{\omega^{2}+c_{0}^{2}}+\sum_{m=1}^{M} \frac{A_{m} c_{m}^{2}}{\omega^{2}+c_{m}^{2}},|\omega| \leq \pi$ and $\theta \in$ $[0, S(0)]$ is the waterfilling parameter [3].

Proof: See [4].

It is important to note that $R^{\mathrm{s}-\mathrm{i}}(D)>R^{\mathrm{c}}(D)$ because it can be shown that the spatial vector of temperature samples $\boldsymbol{T}(t)$ has a non-diagonal correlation matrix (see [4] for details).

\section{2. $R^{\text {st-i }}(D)$ for Spatially-Temporally Independent Coding}

In addition to the constraint of local processing, we now also constrain each sensor to encode the observed time samples separately, disregarding the correlation over time. We call $R^{\mathrm{st}-\mathrm{i}}(D)$ the ratedistortion function for this scenario. Notice that in this case we are constrained to perform quantization only on one temporal sample at a time, and thus, for any optimal entropy-constrained onedimensional quantizer, there is a quantization shaping loss [3]. The following Theorem directly follows:

Theorem 3 For the scenario of spatially and temporally independent coding, the achievable $R^{\text {st-i }}(D)$ function is characterized by:

$(2 M+1) R_{G}(D) \leq R^{s t-i}(D) \leq(2 M+1)\left(R_{G}(D)+\frac{1}{2} \log \left(\frac{\pi e}{6}\right)\right)$

where $R_{G}(D)=\frac{1}{2} \max \left[0, \log \left(\frac{\sigma^{2}}{D}\right)\right], \sigma^{2}=\frac{1}{\pi} \int_{0}^{\pi} S(\omega) d \omega$ is the variance of each sample, and $S(\omega)$ is as given in Theorem 2.

\section{DISTRIBUTED PREDICTION-BASED CODING}

In this section, we consider two prediction based systems. In the first system, we assume that there is a perfect feedback channel from the BS to the sensors, that is, the BS can transmit at an infinite rate. Over time, the system works by sampling at the temporal Nyquist rate, which under our assumptions is 1 . The overall processing is illustrated in Fig. 2. Given an estimate of the temperature vector $\widehat{\boldsymbol{T}}\left(t_{j}\right)=\left[\widehat{T}\left(x_{0}, t_{j}\right), \ldots, \widehat{T}\left(x_{2 M}, t_{j}\right)\right]^{T}$ at time $t_{j}$, the BS computes a temporal prediction $\widetilde{\boldsymbol{T}}\left(t_{j+1}\right)$. Notice that since $\widetilde{\boldsymbol{\beta}}(t)=\frac{2}{2 M+1} \boldsymbol{F}^{T} \widetilde{\boldsymbol{T}}(t)$, we only need to get each prediction $\widetilde{\beta}_{m}\left(t_{j+1}\right), m=0, \ldots, 2 M$. In order to perform this prediction, 


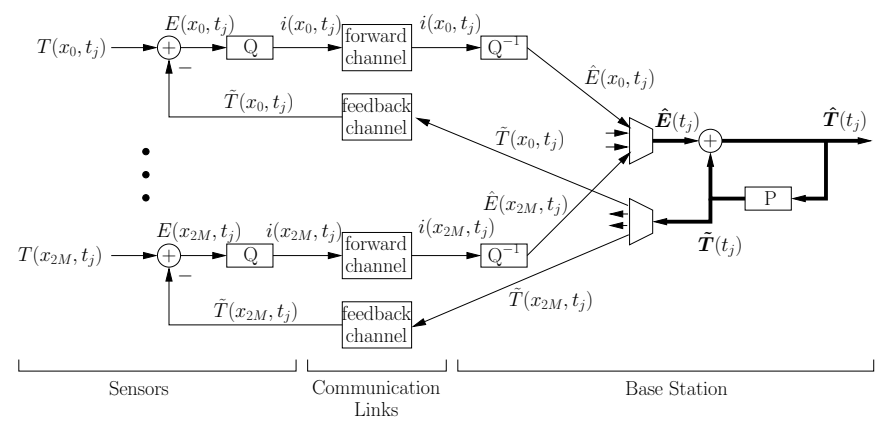

Fig. 2. DPCM based system with feedback from BS.

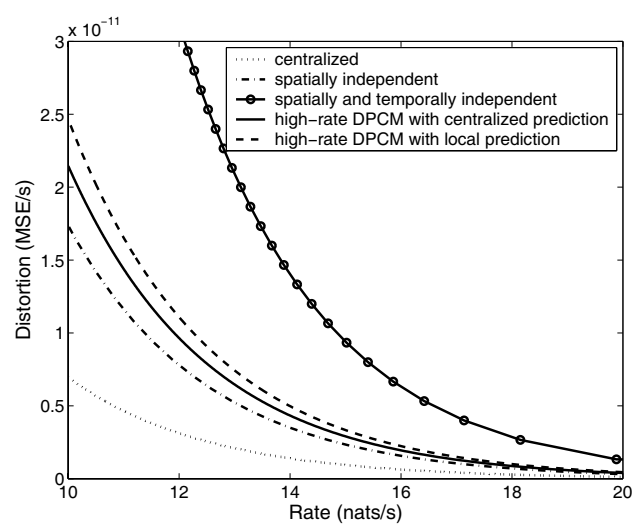

Fig. 3. Comparison at high rates for $G_{m}=1, m=1, \ldots, M$, $L=0.1, M=20$, and the material is silver.

we make use of the Green's Theorem for the heat equation [1], which, applied to our system, establishes that if $\widehat{\boldsymbol{\beta}}\left(t_{j}\right)$ were the actual vector at time $t_{j}$, then $\widetilde{\boldsymbol{T}}\left(t_{j+1}\right)=\boldsymbol{F} \widetilde{\boldsymbol{\beta}}\left(t_{j+1}\right)$, where each component is given by:

$$
\widetilde{\beta}_{m}\left(t_{j+1}\right)=\widehat{\beta}_{m}\left(t_{j}\right) e^{-c_{m}}+\frac{\alpha}{\kappa} e^{-c_{m}} \int_{0}^{1} g_{m}\left(t_{j}+\tau\right) e^{c_{m} \tau} d \tau
$$

The first term in (8) corresponds to a diffusion of the current state $\widehat{\beta}_{m}\left(t_{j}\right)$, and the second one corresponds to an innovation term, which involves the source process $g_{m}(t)$ affecting the system during the interval $\left[t_{j}, t_{j+1}\right]$. Our approach is to consider as prediction the first term, that is, $\widetilde{\beta}_{m}\left(t_{j+1}\right)=\widehat{\beta}_{m}\left(t_{j}\right) e^{-c_{m}}, m=$ $0, \ldots, 2 M$. Once this prediction is performed, the BS obtains the prediction for the temperature vector $\widetilde{\boldsymbol{T}}\left(t_{j+1}\right)=\boldsymbol{F} \widetilde{\boldsymbol{\beta}}\left(t_{j+1}\right)$, and sends to the $n$-th sensor the corresponding predicted value $\widetilde{T}\left(x_{n}, t_{j+1}\right)$. Then, at time $t_{j+1}$, the $n$-th sensor reads the real temperature value $T\left(x_{n}, t_{j+1}\right)$ and quantizes the prediction error $E\left(x_{n}, t_{j+1}\right)=T\left(x_{n}, t_{j+1}\right)-\widetilde{T}\left(x_{n}, t_{j+1}\right)$, getting the quantized output $\widehat{E}\left(x_{n}, t_{j+1}\right)=Q\left(E\left(x_{n}, t_{j+1}\right)\right)$, where $Q$ denotes a uniform scalar quantizer. Next, all the sensors send their quantized prediction errors to the BS, which reconstructs each temperature value as $\widehat{T}\left(x_{n}, t_{j+1}\right)=\widetilde{T}\left(x_{n}, t_{j+1}\right)+\widehat{E}\left(x_{n}, t_{j+1}\right)$. Then, the BS starts performing prediction again and the whole process is repeated. Thus, the overall system behaves as a closed-loop DPCM [5] with centralized prediction at the BS. It can also be shown that the prediction given by the first term in (8) is the opti-

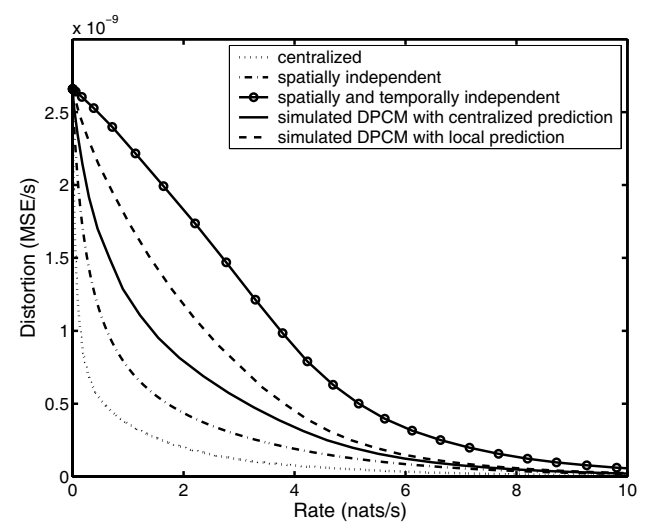

Fig. 4. Comparison at low-to-middle rates for $G_{m}=1, m=$ $1, \ldots, M, L=0.1, M=20$, and the material is silver.

mal 1-tap LMMSE discrete-time prediction filter and that the optimal (infinite-length) prediction filters provides a similar prediction gain (see [4] for details).

In the second system, feedback from BS is not allowed and the $n$-th sensor, independently, performs a closed-loop DPCM system by predicting over time and where the corresponding observed sampled process $T\left(x_{n}, t_{j}\right)$ has a PSD $S(\omega)$ which is given as in Theorem 2, $\forall n=0, \ldots, 2 M$. Again, it can also be shown [4] that the optimal 1-tap LMMSE filter provides a prediction gain very close to that of the optimal filter.

Fig. 3 shows the different analytical rate-distortion curves at high rates (see [4] for details), and Fig. 4 shows the performance obtained by simulation for both DPCM systems. For comparison, we also show $R^{\mathrm{c}}(D), R^{\mathrm{s}-\mathrm{i}}(D)$ and $R^{\mathrm{st}-\mathrm{i}}(D)$ in Fig. 3-4, where in Fig. 4, the curve corresponding to $R^{\text {st-i }}(D)$ has been also obtained by simulation with the same uniform scalar quantizer used for the DPCM systems. As expected, the local DPCM system provides better performance than $R^{\mathrm{st}-\mathrm{i}}(D)$. On the other hand, the DPCM system with centralized prediction, since it makes use of temporal information from all the sensors, is superior to the local DPCM system. However, the DPCM system with centralized prediction is still inferior to $R^{s-\mathrm{i}}(D)$ which involves infinite complexity (although the difference is less than the quantization shaping gain).

Remark: In [4], it is shown that it is possible to design an appropriate (Wyner-Ziv) nested code by using as side information the (spatial-temporal) physics-based predictions made at the BS, so that each sensor only needs to transmit cosets. Notice that each $T\left(x_{n}, t_{j}\right)$ is correlated with the predicted vector $\widetilde{\boldsymbol{T}}\left(t_{j}\right)$. Thus, this is a Wyner-Ziv problem with multiple side-information $(2 M+1$ predictions). This scheme avoids the need of feedback from BS.

\section{REFERENCES}

[1] J. V. Beck, K. D. Cole, A. Haji-Sheikh and B. Litkouhi. Heat Conduction Using Green's Functions, W. J. Minkowycz and E. M. Sparrow, Eds., Hemisphere Publishing Corporation, 1992.

[2] S. Pradhan. Distributed Source Coding Using Syndromes (DISCUS). PhD Thesis, University of California, Berkeley, 2001.

[3] T. Berger. Rate Distortion Theory. Prentice-Hall, 1971.

[4] B. Beferull-Lozano, R. L. Konsbruck and M. Vetterli. Rate-Distortion and Communication for Physics Based Distributed Sensing. In preparation to be submitted to IEEE Trans. on Inf. Theory.

[5] N. S. Jayant and P. Noll. Digital Coding of Waveforms. 1984. 